\title{
Fortalezas Personales y Hábitos de Salud en Estudiantes Universitarios de Ciencias del Movimiento Humano
}

\author{
Character Strengths and Health Habits in College Students of Human Movement Sciences
}

\author{
Kristy Barrantes-Brais, Pedro Ureña Bonilla y Juan Carlos Gutiérrez \\ Vargas \\ Escuela de Ciencias del Movimiento Humano y Calidad de Vida, \\ Universidad Nacional, Costa Rica \\ kristy.barrantes.brais@una.cr
}

\section{Resumen}

\begin{abstract}
El propósito de este estudio fue caracterizar y relacionar las fortalezas personales y los hábitos de salud en estudiantes universitarios de carreras de Ciencias del Movimiento Humano. Un total de 234 estudiantes (137 hombres y 97 mujeres) brindaron información sobre fortalezas personales, hábitos de sueño y consumo de sustancias. Se analizaron diferencias entre grupos y correlaciones entre variables. Los resultados sugieren que las fortalezas predominantes son gratitud, curiosidad, integridad, bondad y liderazgo; se encuentran diferencias según género y carrera. Predominan los hábitos saludables, los cuales se asocian con algunas fortalezas. Los estudiantes presentan fortalezas que denotan preocupación por los demás y posibilidades de expresar y ser sensibles a las emociones. Los hábitos saludables y su relación con algunas fortalezas parecen evidenciar coherencia con las características de la unidad académica a la que pertenecen.
\end{abstract}

Palabras Claves: población estudiantil, psicología positiva, sueño, consumo de sustancias

\begin{abstract}
The purpose of this study was to define and relate character strengths and health habits in a sample of college students enrolled in Human Movement Sciences careers. A total of 234 students (137 males and 97 females) provided information on character strengths and sleep and substance use habits. Group differences and variable correlations were analyzed. The results suggest that the main strengths are: gratitude, curiosity, authenticity, kindness and leadership. There are differences when comparing careers and genders. Healthy habits prevail and correlate with some strengths. Strengths in this sample show that students tend to care for others and are able to express and be sensitive to emotions. Healthy habits and their relation with character strengths appear consistent with the qualities of the school they belong to.
\end{abstract}

Key Words: student population, positive psychology, sleep, substance use 


\section{Introducción}

Históricamente las universidades han creado culturas enfocadas en el rendimiento y la excelencia (Oades, Robinson, Green y Spence, 2011). Esta visión promueve la búsqueda de soluciones a obstáculos que impiden un desempeño adecuado según los parámetros establecidos, es decir, se constituye en un enfoque basado en las deficiencias (Roberts, Brown, Johnson y Reinke, 2002) desde el cual lo importante ha sido estudiar a las poblaciones estudiantiles y los entornos educativos centralizándose en aspectos relacionados con el rendimiento académico y las capacidades para cumplir con parámetros de logro (Pedrals, Rigotti y Bitrán, 2011; Seligman, Ernst, Gillham, Reivich y Linkins, 2009). Esta visión coincide con las formas tradicionales de conceptualizar al ser humano desde la ciencia psicológica, con énfasis en la patología y las problemáticas que le impiden desarrollarse plenamente (Maddux, 2002). Como respuesta a esta visión, a finales de los años 90 surge una propuesta para volver la mirada a aquellos elementos que, según sus precursores, "hacen que la vida merezca ser vivida" (Seligman y Csikszentmihalyi, 2000), favoreciendo así el funcionamiento humano óptimo (Linley y Joseph, 2004).

Desde esta visión alternativa se promueve el estudio de las fortalezas personales, entendidas como capacidades naturales que tiene todo ser humano, que se manifiestan en la forma de pensar, sentir, actuar y posibilitan el funcionamiento y desempeño de las personas (Linley y Harrington, 2006). Park y Peterson (2009) hacen énfasis en el hecho de que las fortalezas constituyen características positivas que existen en diferentes grados, como en un continuum, y que se hacen evidentes en los pensamientos, sentimientos y hábitos de las personas. Estos autores proponen una clasificación de 24 fortalezas personales (FP) agrupadas en seis virtudes: la Sabiduría y el Conocimiento, el Coraje, la Humanidad, la Justicia, la Templanza y la Trascendencia. Bajo este modelo, se argumenta que las FP desencadenan procesos o mecanismos psicológicos específicos que definen y dan contenido a las virtudes (Park y Peterson, 2009). La Sabiduría y el Conocimiento engloban: la creatividad, la curiosidad, la apertura mental, el amor por el saber y la perspectiva. El Coraje está constituido por la valentía, la persistencia, la integridad y la vitalidad. La Humanidad se caracteriza por el amor, la bondad y la inteligencia social. La Justicia es definida por la ciudadanía, la imparcialidad y el liderazgo. La Templanza la integran: la clemencia/misericordia, la humildad/modestia, la prudencia y la autorregulación. La Trascendencia está compuesta por la apreciación de la belleza y la excelencia, la gratitud, la esperanza, el humor y la espiritualidad (Park, Peterson y Seligman, 2006).

Con base en este modelo se han realizado numerosos estudios en los que se comparan los perfiles de fortalezas entre diversos países, regiones y culturas (Brdar y Kashdan, 2010; Leontopoulou y Triliva, 2012; Park et al., 2006). Importantes aportes ha brindado el análisis de la relación entre las fortalezas personales y constructos como el bienestar subjetivo y la calidad de vida (Gillham et al., 2011; Giménez Hernández, 2010; Proctor, Maltby y Linley, 2011), el éxito académico (Cosentino y Castro Solano, 2012; Lounsbury, Fisher, Levy y Welsh, 2009) y el consumo de sustancias (Logan, Kilmer y Marlatt, 2010). Fortalezas como la gratitud, la esperanza, la vitalidad, la curiosidad y el amor se asocian consistentemente de manera positiva con indicadores de bienestar subjetivo; mientras que la perseverancia, el amor, la gratitud, la esperanza y la perspectiva, han mostrado ser buenos predictores del éxito académico (Leontopoulou y Triliva, 2012; Park et al., 2006). 
En general se plantea que las fortalezas personales se vinculan a indicadores positivos de salud física (Seligman, 2008; Vázquez, Hervás, Rahona, y Gómez, 2009; Veenhoven, 2008). Asimismo, la práctica de la gratitud se relaciona con la reducción de síntomas físicos, con estrategias de afrontamiento adaptativas y con más tiempo dedicado al ejercicio físico, lo que podría vincularse a una mayor sensación de bienestar y funcionamiento social. Incluso en personas con enfermedad neuromuscular, la práctica de la gratitud se vincula con aumentos en la sensación general de bienestar (Emmons y McCullough, 2003).

Bajo el supuesto de que las fortalezas tienen un componente conductual, no cabe duda de que el ejercicio de las características positivas personales en forma de hábitos contribuye con la salud física y emocional de las personas (Oblitas, 2008). También se ha reportado que la participación de los jóvenes en experiencias que fortalezcan el carácter y la valentía se asocia con aumentos en la satisfacción con la vida (Proctor et al., 2011). Fortalezas como la bondad y el trabajo en equipo se relacionan con la reducción de síntomas depresivos; mientras que fortalezas trascendentales, como la espiritualidad, se vinculan con niveles altos de satisfacción con la vida (Gillhan et al., 2011).

En general, se afirma que las fortalezas prevalentes en los seres humanos son en orden descendente: la bondad, la justicia, la honestidad, la gratitud y el juicio; mientras que las de menor prevalencia son la prudencia, la modestia y la autorregulación (Park et al., 2006). Adultos jóvenes norteamericanos con edades entre los 18-28 años, comparados con homólogos japoneses, mostraron perfiles similares en cuanto a fortalezas personales. Los valores más altos correspondieron a la bondad, el humor y el amor; como contraste, los valores más bajos se registraron en relación con la prudencia, la modestia y la autorregulación. Asimismo, las mujeres, en comparación con los hombres, mostraron valores significativamente más altos en bondad, valentía y creatividad (Shimai, Otake, Park, Peterson y Seligman, 2006).

Además, se argumenta que la esperanza, la bondad, la inteligencia social, la autorregulación y la perspectiva reducen los efectos negativos del estrés traumático (Park y Peterson, 2009; Park et al., 2006). También se ha reportado que fortalezas como la esperanza, el ánimo y el liderazgo tienen una relación inversamente proporcional con los niveles de ansiedad y depresión (Park y Peterson, 2008).

De igual forma, se ha planteado que el reconocimiento y puesta en práctica de fortalezas personales podría asociarse también con la presencia de indicadores positivos de salud física (Seligman, 2008; Vázquez, Hervás, Rahona, y Gómez, 2009; Veenhoven, 2008). Existen algunas explicaciones psicobiológicas para esta relación (Chida y Steptoe, 2008; Kiecolt-Glaser, McGuire, Robles y Glaser, 2002; Steptoe, Dockray y Wardle, 2009); ahora bien, si se considera que las fortalezas tienen un componente conductual, podría plantearse que el ejercicio de las características positivas personales en forma de hábitos contribuiría al funcionamiento óptimo, incluyendo la salud física y emocional. Siguiendo a Oblitas (2008), se reconoce que "nuestra salud está profundamente influenciada por nuestra conducta, pensamientos y relaciones sociales (...) nuestra conducta tiene un efecto inmenso en nuestra salud" (p.11). 
El estudio de los hábitos de salud se circunscribe a su naturaleza como componente de los estilos de vida de las personas (Sanabria, González y Urrego, 2007). Los estilos de vida comprenden el conjunto de pautas y hábitos comportamentales cotidianos de una persona o aquellos patrones de conducta individuales que demuestran cierta consistencia en el tiempo, bajo condiciones más o menos constantes y que pueden constituirse en dimensiones de riesgo o de seguridad. Dependiendo de su naturaleza, incluye entre sus componentes el factor cognitivo. Específicamente, los estilos de vida saludables incluyen conductas de salud, patrones de conducta, creencias, conocimientos, hábitos y acciones de las personas para mantener, restablecer y mejorar su salud (Arrivillaga y Salazar, 2005; Roth, 1990).

Estudios previos han señalado que los estudiantes universitarios se encuentran en riesgo de presentar hábitos no saludables de sueño (Gaultney, 2010; Sing y Wong, 2010) y de consumo de sustancias como alcohol, tabaco y otras drogas (Guerra de Andrade et al., 2012; Jiménez-Muro, Beamonte, Marqueta, Gargallo y Nerín de la Puerta, 2009; Primack et al., 2012). Otras investigaciones presentan resultados en los que los estudiantes universitarios reportan hábitos saludables (Grimaldo, 2012; Lema et al., 2009). Desde el movimiento de universidades promotoras de la salud se ha reconocido la importancia de generar conocimiento y realizar intervenciones en áreas que involucren los estilos de vida de la comunidad universitaria (Lange y Vio, 2006; Muñoz y Cabieses, 2008).

La evidencia es clara en cuanto al rol que juegan las fortalezas personales en la salud física y emocional de las personas. La caracterización de grupos poblacionales a partir de las fortalezas personales brindará información valiosa para implementar intervenciones direccionadas hacia su fortalecimiento, máxime si se trata de futuros profesionales en el campo de la educación, quienes tendrán la tarea de coadyuvar en el proceso conducente a la interiorización de valores y al desarrollo integral de niños, niñas y jóvenes. Sobre esta temática se ha investigado poco en Costa Rica, de manera que se acusa un vacío importante de conocimiento en materia de fortalezas y valores. Con base en la evidencia reportada, y conscientes de la importancia que tiene el estudio de las fortalezas personales, el propósito de esta investigación fue caracterizar y relacionar las fortalezas personales y los hábitos de salud en estudiantes costarricenses de Ciencias del Movimiento Humano.

\section{Metodología}

\section{Participantes.}

Se contó con la participación de 234 estudiantes de la Escuela de Ciencias del Movimiento Humano y Calidad de Vida de la Universidad Nacional (CIEMHCAVI), 137 hombres y 97 mujeres, con edades promedio de 22,8 $\pm 4,8$ años y 23,3 $\pm 4,4$ años, respectivamente. Se pretendió recolectar información de la totalidad de la población estudiantil de la Escuela CIEMHCAVI (según se indica en el procedimiento), la tasa de respuesta fue del $70 \%$. El criterio de inclusión fue encontrarse matriculado en al menos un curso de la carrera correspondiente en el II ciclo 2012. La muestra se distribuyó de la siguiente forma: Bachillerato en Educación Física, Deporte y Recreación (45 \%), Bachillerato en Promoción de la Salud Física (28 \%), Licenciatura en Ciencias del Deporte (11\%) y Maestría en Salud Integral y Movimiento Humano (16\%). 


\section{Instrumentos.}

Se utilizó el Inventario de Virtudes y Fortalezas [IVyF] (Cosentino y Castro Solano, 2008). Este es un autoinforme mediante el cual se estudian 24 fortalezas humanas (Park y Peterson, 2009; Peterson y Seligman, 2003). El IVyF se desarrolló como una opción breve del VIA-IS (Peterson y Park, 2004) y mantiene características psicométricas similares (Cosentino y Castro Solano, 2012). El IVyF está compuesto por 24 ítems bipolares (uno para cada fortaleza), con cinco opciones de respuesta tipo Likert. Se solicita a los encuestados que indiquen en qué grado se parecen más a una de dos auto-descripciones propuestas, una con presencia de una fortaleza del carácter y la otra con ausencia. La puntuación de cada ítem va de 1 (Soy muy parecido a la $1^{\text {a }}$ persona) a 5 (Soy muy parecido a la $2^{\text {a }}$ persona). A mayor puntaje, mayor presencia de la fortaleza. Al IVyF se le reporta una estabilidad test-retest que oscila entre 0.72 a 0.92 (Cosentino, 2009; Cosentino y Castro Solano, 2012).

Asimismo, se aplicó el Cuestionario de prácticas y creencias sobre estilos de vida (Arrivillaga, Salazar y Correa, 2003), el cual evalúa aspectos relacionados con el sueño y el consumo de alcohol, tabaco y otras drogas. Para cada componente se presentan 10 enunciados que se responden de acuerdo a la frecuencia de su puesta en práctica (siempre, frecuentemente, algunas veces y nunca). A mayor puntaje, más saludables serán los hábitos. Los valores promedio permiten establecer categorías de calificación de los hábitos o prácticas: muy saludable, saludable, poco saludable y no saludable (Arrivillaga y Salazar, 2005; Arrivillaga et al., 2003). Se reporta una consistencia interna de $\alpha=0,873$.

\section{Procedimiento.}

Los instrumentos se aplicaron a todos los estudiantes presentes durante lecciones regulares de todas las carreras de Ciencias del Movimiento Humano, distribuidas en mañanas, tardes y noches en el periodo entre agosto y setiembre del 2012. Se coordinó previamente con la administración y los profesores a cargo. La aplicación de los cuestionarios estuvo a cargo de asistentes graduadas de carreras de Ciencias del Movimiento Humano con experiencia en recolección de datos. En el encuadre (verbal y por escrito) se le indicó a la población estudiantil que la participación era voluntaria y se garantizó la confidencialidad en el uso y procesamiento de la información.

\section{Análisis estadístico.}

Se calcularon valores promedios con las respectivas desviaciones típicas, $t$ de Student para grupos independientes con el propósito de realizar comparaciones según género, análisis de varianza para el contraste de promedios en función del nivel y la carrera, así como la prueba HSD de Tukey para análisis post hoc. La correlación entre las fortalezas y hábitos de salud se calculó mediante la prueba $r$ de Pearson. Asimismo, el contraste de proporciones se realizó mediante la prueba $\mathrm{Chi}^{2}$ de Pearson. Los datos fueron procesados mediante el programa PASW Statistics 18 y todas las decisiones estadísticas se tomaron considerando un nivel alfa $\leq 0,05$. 


\section{Resultados}

En general, los valores promedio más altos corresponden a las fortalezas: gratitud, curiosidad, integridad, bondad y liderazgo; mientras que los promedios más bajos se registran en relación con las fortalezas: clemencia, ciudadanía, autorregulación, humildad y amor por el saber (ver tabla 1).

Tabla 1

Valores promedio en general y según sexo para las fortalezas personales

\begin{tabular}{|c|c|c|c|c|}
\hline Fortalezas & General & Hombres & Mujeres & Sig. \\
\hline Gratitud & $4.49 \pm 0.81$ & $4.43 \pm 0.88$ & $4.57 \pm 0.71$ & 0.89 \\
\hline Curiosidad & $4.36 \pm 0.77$ & $4.35 \pm 0.78$ & $4.36 \pm 0.76$ & 0.97 \\
\hline Integridad & $4.28 \pm 0.90$ & $4.22 \pm 0.93$ & $4.35 \pm 0.86$ & 0.27 \\
\hline Bondad & $4.28 \pm 0.74$ & $4.21 \pm 0.80$ & $4.36 \pm 0.64$ & 0.15 \\
\hline Liderazgo & $4.22 \pm 0.91$ & $4.26 \pm 0.87$ & $4.19 \pm 0.92$ & 0.57 \\
\hline Vitalidad & $4.15 \pm 0.85$ & $4.07 \pm 0.84$ & $4.25 \pm 0.84$ & 0.10 \\
\hline Perspectiva & $4.12 \pm 0.83$ & $4.10 \pm 0.87$ & $4.15 \pm 0.78$ & 0.62 \\
\hline Creatividad & $4.10 \pm 0.90$ & $4.07 \pm 0.91$ & $4.15 \pm 0.90$ & 0.50 \\
\hline Apertura mental & $4.08 \pm 0.84$ & $4.08 \pm 0.82$ & $4.08 \pm 0.87$ & 0.95 \\
\hline Persistencia & $4.05 \pm 1.04$ & $4.02 \pm 1.02$ & $4.07 \pm 1.06$ & 0.75 \\
\hline Imparcialidad & $4.04 \pm 1.04$ & $4.05 \pm 1.02$ & $4.01 \pm 1.07$ & 0.72 \\
\hline Inteligencia social & $4.03 \pm 0.99$ & $4.05 \pm 1.04$ & $4.00 \pm 0.92$ & 0.69 \\
\hline Espiritualidad & $4.00 \pm 0.93$ & $3.89 \pm 1.04$ & $4.13 \pm 0.73$ & 0.05 \\
\hline Prudencia & $3.93 \pm 0.89$ & $3.89 \pm 0.90$ & $4.00 \pm 0.86$ & 0.38 \\
\hline Apreciación & $3.88 \pm 0.92$ & $3.76 \pm 0.96$ & $4.04 \pm 0.85$ & 0.02 \\
\hline Amor & $3.82 \pm 1.10$ & $3.67 \pm 1.11$ & $4.03 \pm 1.07$ & 0.01 \\
\hline Esperanza & $3.82 \pm 0.94$ & $3.84 \pm 0.96$ & $3.78 \pm 0.92$ & 0.61 \\
\hline Valentía & $3.77 \pm 0.97$ & $3.80 \pm 1.02$ & $3.72 \pm 0.92$ & 0.53 \\
\hline Humor & $3.71 \pm 1.02$ & $3.70 \pm 1.08$ & $3.71 \pm 0.94$ & 0.93 \\
\hline Clemencia & $3.67 \pm 0.99$ & $3.63 \pm 1.02$ & $3.72 \pm 0.97$ & 0.51 \\
\hline Ciudadanía & $3.56 \pm 0.95$ & $3.48 \pm 1.02$ & $3.67 \pm 0.83$ & 0.12 \\
\hline Autorregulación & $3.44 \pm 1.13$ & $3.45 \pm 1.15$ & $3.43 \pm 1.11$ & 0.90 \\
\hline Humildad & $3.37 \pm 1.12$ & $3.27 \pm 1.18$ & $3.53 \pm 1.01$ & 0.07 \\
\hline Amor por el saber & $3.18 \pm 1.06$ & $3.00 \pm 1.13$ & $3.43 \pm 0.92$ & 0.002 \\
\hline
\end{tabular}

Nota: Diferencias significativas $(p<.05)$ en negrita

Las mujeres, comparadas con los hombres, muestran valores promedio significativamente más altos en las fortalezas apreciación, amor y amor por el saber. La comparación de fortalezas por nivel no mostró diferencias significativas $(p>0,05)$. 
El análisis comparativo por carrera (ver tabla 2) mostró diferencias significativas en cuanto a algunas fortalezas. Las comparaciones usando la prueba post hoc HSD de Tukey mostraron diferencias significativas en apertura mental entre el Bachillerato en Educación Física, Deporte y Recreación (EF) y la Maestría en Salud Integral y Movimiento Humano (MSIMH), siendo este posgrado en el que los estudiantes reportan niveles más altos. En cuando al liderazgo, los estudiantes de las carreras de EF y Licenciatura en Ciencias del Deporte (Licenciatura) presentan puntajes significativamente más altos en comparación con los del Bachillerato en Promoción de la Salud Física (Promoción). En la fortaleza persistencia, se encuentran diferencias entre estudiantes de Promoción y de la MSIMH (los últimos presentan puntuaciones mayores en esta fortaleza). En el caso de prudencia, los estudiantes de la MSIMH se ubican por encima de los que cursan las carreras de EF y Licenciatura. Por último, los puntajes correspondientes a las carreras Promoción y MSIMH en amor por el saber son significativamente más altos que las de EF.

Tabla 2

Clasificación de las Fortalezas personales según la carrera

\begin{tabular}{|c|c|c|c|c|c|c|}
\hline Fortalezas & $\begin{array}{c}\text { Educación } \\
\text { Física } \\
\end{array}$ & Promoción & Licenciatura & MSIMH & $F$ & Sig. \\
\hline Gratitud & $1(4.49 \pm 0.83)$ & $1(4.43 \pm 0.80)$ & $2(4.53 \pm 0.85)$ & $1(4.59 \pm 0.79)$ & 0.335 & 0.80 \\
\hline Liderazgo & $2(4.35 \pm 0.78)$ & $12(3.96 \pm 0.99)$ & $1(4.53 \pm 0.90)$ & $11(4.05 \pm 1.02)$ & 3.927 & 0.009 \\
\hline Curiosidad & $3(4.35 \pm 0.78)$ & $4(4.24 \pm 0.81)$ & $3(4.42 \pm 0.85)$ & $2(4.56 \pm 0.55)$ & 1.432 & 0.23 \\
\hline Bondad & $4(4.31 \pm 0.78)$ & $3(4.30 \pm 0.72)$ & $5(4.38 \pm 0.69)$ & $10(4.08 \pm 0.68)$ & 1.150 & 0.33 \\
\hline Integridad & $5(4.26 \pm 0.92)$ & $2(4.40 \pm 0.86)$ & $14(3.88 \pm 1.07)$ & $4(4.40 \pm 0.76)$ & 2.314 & 0.07 \\
\hline Vitalidad & $6(4.21 \pm 0.80)$ & $10(4.03 \pm 0.91)$ & $9(4.15 \pm 0.88)$ & $9(4.21 \pm 0.85)$ & 0.671 & 0.57 \\
\hline Creatividad & $7(4.16 \pm 0.90)$ & $13(3.96 \pm 0.95)$ & $4(4.40 \pm 0.64)$ & $12(4.02 \pm 0.98)$ & 1.595 & 0.19 \\
\hline Inteligencia social & $8(4.09 \pm 0.98)$ & $11(3.98 \pm 1.01)$ & $12(3.92 \pm 1.05)$ & $13(4.00 \pm 1.00)$ & 0.301 & 0.82 \\
\hline Perspectiva & $9(4.09 \pm 0.84)$ & $8(4.07 \pm 0.93)$ & $10(4.15 \pm 0.83)$ & $6(4.27 \pm 0.65)$ & 0.485 & 0.69 \\
\hline Persistencia & $10(4.04 \pm 1.02)$ & $16(3.73 \pm 1.25)$ & $7(4.26 \pm 0.77)$ & $3(4.45 \pm 0.64)$ & 4.384 & 0.005 \\
\hline Imparcialidad & $11(4.04 \pm 1.00)$ & $9(4.04 \pm 1.11)$ & $16(3.76 \pm 1.14)$ & $8(4.21 \pm 0.97)$ & 0.933 & 0.42 \\
\hline Espiritualidad & $12(3.93 \pm 1.03)$ & $6(4.09 \pm 0.87)$ & $11(4.03 \pm 0.77)$ & $15(4.00 \pm 0.84)$ & 0.402 & 0.75 \\
\hline Apertura mental & $13(3.92 \pm 0.87)$ & $5(4.12 \pm 0.86)$ & $8(4.26 \pm 0.66)$ & $5(4.36 \pm 0.76)$ & 3.082 & 0.02 \\
\hline Humor & $14(3.86 \pm 1.00)$ & $18(3.73 \pm 1.01)$ & $22(3.46 \pm 0.90)$ & $24(3.37 \pm 1.13)$ & & 0.05 \\
\hline Esperanza & $15(3.84 \pm 0.90)$ & $17(3.73 \pm 1.04)$ & $20(3.65 \pm 0.89)$ & $14(4.00 \pm 0.94)$ & 0.891 & 0.44 \\
\hline Prudencia & $16(3.79 \pm 0.94)$ & $7(4.09 \pm 0.72)$ & $18(3.65 \pm 1.16)$ & $7(4.27 \pm 0.65)$ & 4.344 & 0.005 \\
\hline Amor & $17(3.78 \pm 1.14)$ & $14(3.93 \pm 1.10)$ & $15(3.88 \pm 1.05)$ & $20(3.69 \pm 1.09)$ & 0.464 & 0.70 \\
\hline Apreciación & $18(3.78 \pm 0.90)$ & $15(3.86 \pm 0.94)$ & $6(4.30 \pm 0.78)$ & $16(3.94 \pm 0.99)$ & 2.328 & 0.07 \\
\hline Valentía & $19(3.75 \pm 0.94)$ & $21(3.63 \pm 1.13)$ & $13(3.92 \pm 0.97)$ & $17(3.94 \pm 0.70)$ & 1.065 & 0.36 \\
\hline Clemencia & $20(3.71 \pm 1.01)$ & $19(3.72 \pm 1.05)$ & $23(3.34 \pm 1.01)$ & $19(3.70 \pm 0.84)$ & 1.046 & 0.37 \\
\hline Ciudadanía & $21(3.54 \pm 0.98)$ & $20(3.64 \pm 0.95)$ & $21(3.52 \pm 0.91)$ & $22(3.51 \pm 0.93)$ & 0.227 & 0.87 \\
\hline Humildad & $22(3.25 \pm 1.12)$ & $23(3.46 \pm 1.09)$ & $19(3.65 \pm 1.12)$ & $23(3.37 \pm 1.18)$ & 1.041 & 0.37 \\
\hline Autorregulación & $23(3.22 \pm 1.17)$ & $22(3.55 \pm 1.11)$ & $17(3.72 \pm 1.17)$ & $18(3.72 \pm 0.91)$ & 2.747 & 0.05 \\
\hline Amor por el saber & $24(2.87 \pm 1.03)$ & $24(3.41 \pm 0.99)$ & $24(3.30 \pm 1.08)$ & $21(3.54 \pm 1.09)$ & 5.691 & 0.001 \\
\hline
\end{tabular}

Notas. Medias y desviaciones estándar entre paréntesis. Educación Física= Bachillerato en Educación Física, Deporte y Recreación, Promoción= Bachillerato en Promoción de la Salud Física, Licenciatura= Licenciatura en Ciencias del Deporte, MSIMH= Maestría en Salud Integral y Movimiento Humano, Apreciación = Apreciación de la belleza y la excelencia. Números antes de los paréntesis indican posición ordinal de las fortalezas en cada carrera. Diferencias significativas $(p<.05)$ en negrita. En cursiva y negrita los datos de los grupos entre los cuales se encontraron diferencias significativas. 
Para el reporte de resultados de los hábitos de consumo de sustancias y sueño se utilizan las categorías de clasificación propuestas por Arrivillaga et al. (2003): Muy saludable, Saludable, Poco Saludable y No Saludable. Estas categorías se establecieron en términos de la frecuencia de las prácticas ejecutadas. En cuanto a los hábitos relacionados con el consumo de alcohol y otras drogas, el $34 \%$ de los estudiantes encuestados se ubica en la categoría de saludable y el $66 \%$ en la categoría de muy saludable. La diferenciación por sexo mostró para el caso de los hombres que un $37 \%$ reportan hábitos saludables y un $63 \%$ hábitos muy saludables. En contraste, las mujeres reportan un $29 \%$ hábitos saludables y un $71 \%$ hábitos muy saludables. La forma en que se distribuyen los porcentajes de hábitos saludables y no saludables entre hombres y mujeres fue similar $\left.\left[\mathrm{Chi}^{2}=1,75 ; p>0.05\right)\right]$. Mientras que en los hábitos de sueño se encontró un $16 \%$ clasificado como no saludable, un $72 \%$ como saludable y un $12 \%$ como muy saludable. Un $12 \%$ de los hombres reporta hábitos poco saludables, un $77 \%$ hábitos saludables y un $11 \%$ hábitos muy saludables. En el caso de las mujeres se reporta en un $21 \%$ hábitos poco saludables, $66 \%$ hábitos saludables y $13 \%$ hábitos muy saludables. Se encontraron diferencias significativas en la distribución de hábitos saludables y no saludables de sueño entre hombres y mujeres $\left.\left[\mathrm{Chi}^{2}=4,21 ; p<0.05\right)\right]$. Las mujeres evidencian mayores dificultades que los hombres en relación con los hábitos de sueño.

El análisis de la relación entre fortalezas y hábitos mostró correlaciones significativas en varias de las dimensiones estudiadas (figuras 1 y 2). Las fortalezas humanas relacionadas con los hábitos de sueño son diferentes a las fortalezas que se relacionan con el consumo de alcohol y otras drogas.

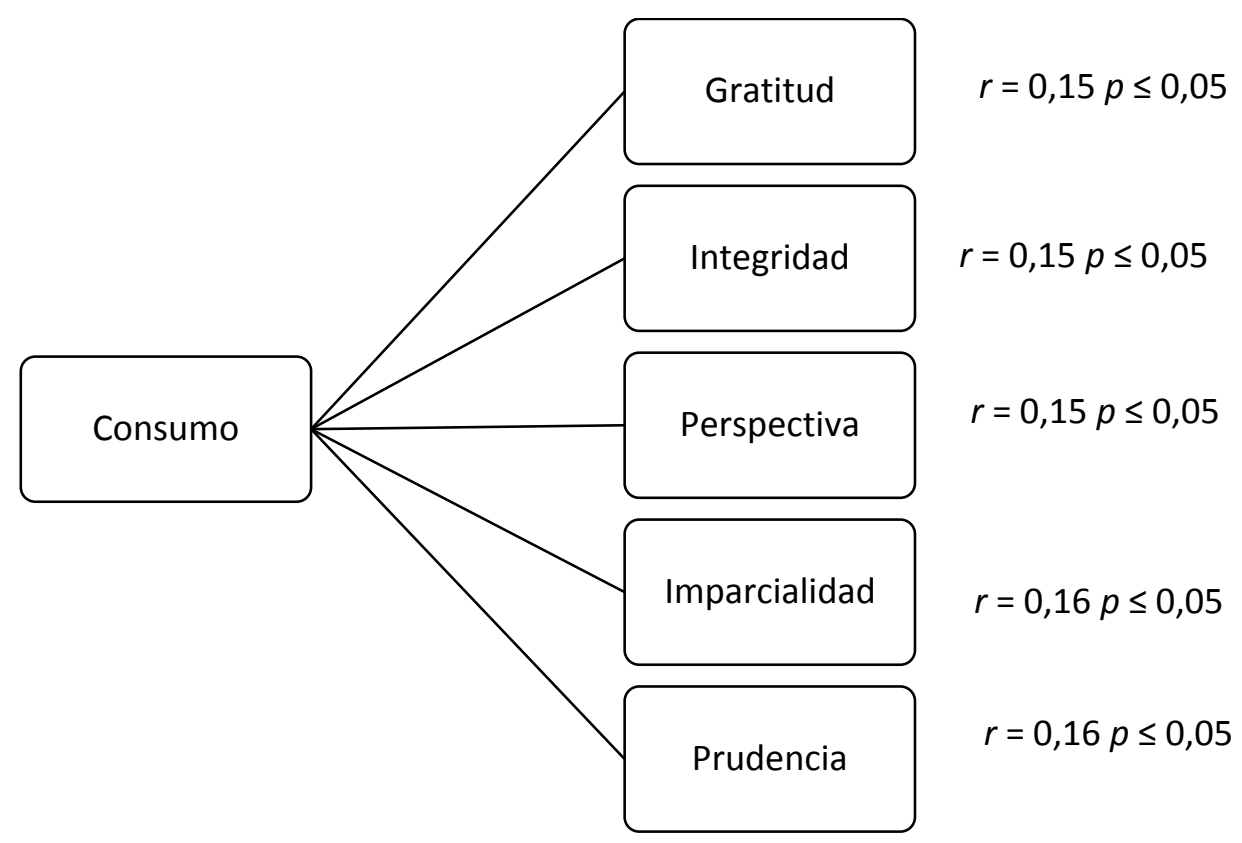

Figura 1. Correlaciones entre hábitos de consumo y fortalezas humanas 


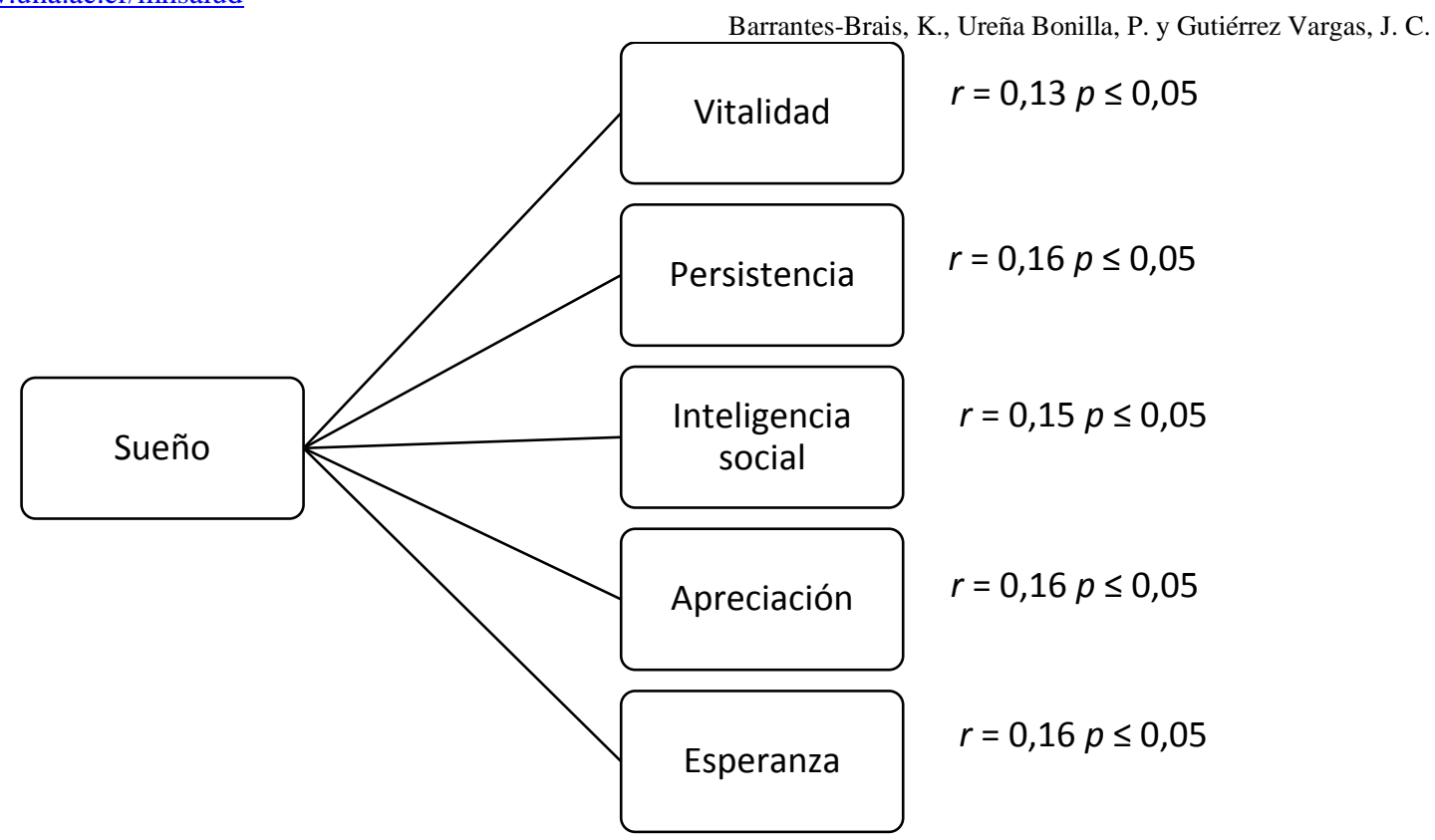

Figura 2. Correlaciones entre hábitos de sueño y fortalezas humanas

\section{Discusión}

Las fortalezas personales que aparecen como más relevantes para los estudiantes de CIEMHCAVI son la gratitud, la curiosidad, la integridad, la bondad y el liderazgo, las cuales representan expresiones prácticas de las virtudes trascendencia, sabiduría, coraje, humanidad y justicia, respectivamente, según la clasificación de Peterson y Seligman (2003). Entre las fortalezas menos predominantes se encuentran las relacionadas con la templanza: autorregulación, humildad/modestia y clemencia/misericordia. Se identifican algunas diferencias por género y por carrera. Los resultados correspondientes a los hábitos de salud muestran una población predominantemente saludable y algunas fortalezas personales se asocian con prácticas saludables.

El interés por el estudio científico de las fortalezas humanas se centra en los potenciales, los motivos y las capacidades que hacen que una gran parte de las personas se adapte exitosamente en la mayoría de las circunstancias de la vida (Sheldon y King, 2001). Es decir, las fortalezas cumplen un papel fundamental en la existencia humana. La gratitud y la curiosidad, que aparecen como relevantes para la muestra en estudio, se han asociado de manera consistente con una elevada satisfacción con la vida e indicadores de bienestar y felicidad (Brdrar y Kashdan, 2010; Macaskill, 2012; Park y Peterson, 2008; Park, Peterson y Seligman, 2004; Peterson, Ruch, Beermann, Park y Seligman, 2007; Shimai et al., 2006). La bondad se ha asociado con bienestar (Proctor et al., 2011), menos síntomas de depresión (Gillham et al., 2011), afecto positivo y fortalecimiento de la conducta prosocial (Giménez Hernández, 2010), y es también una de las fortalezas más prevalentes en diversas poblaciones (Park et al., 2006; Shimai et al., 2006). En esta población podría estarse presentando lo que previamente han descrito Peterson et al. (2007), al plantear que una vida satisfactoria se relaciona con el ejercicio o puesta en práctica de las fortalezas personales. Siguiendo a Aristóteles, los autores plantean que es posible que la satisfacción con la vida resulte intrínseca a vivir de manera coherente con ciertas fortalezas personales. 
La puesta en práctica de las fortalezas en muchas ocasiones se ve facilitada en las relaciones con los demás. En este sentido resulta interesante notar que, con excepción de la curiosidad, las fortalezas más relevantes para los estudiantes en esta investigación los ubican como personas que tienden a centrarse en los demás más que en sí mismos, según los planteamientos de Peterson, 2006. Se ha propuesto también que algunas fortalezas se acercan más al "corazón" (en tanto implican expresiones emocionales) y otras se relacionan más con la "mente" (en las que el intelecto tiene predominancia) (Peterson, 2006). De acuerdo con este planteamiento, los resultados de este estudio muestran estudiantes más cercanos a las "fortalezas del corazón" (como gratitud y bondad). La integridad es la fortaleza relevante en esta población que más se aproxima a las "fortalezas de la mente". Así, el grupo en estudio estaría compuesto por estudiantes preocupados por los demás y con posibilidades de expresar y ser sensibles a las emociones, lo cual resulta significativo en carreras que requieren de habilidades para el trabajo con personas y cuyas labores se verán potenciadas en tanto se logren establecer conexiones con las poblaciones meta.

La gratitud se ubica como la fortaleza más arraigada en los estudiantes de CIEMHCAVI. Con la excepción de la carrera de Licenciatura en Ciencias del Deporte, donde el liderazgo se ubica como la fortaleza más sobresaliente, en el resto de las carreras y en los análisis por género y nivel, la gratitud predomina. Esta fortaleza implica ser consciente y agradecido por las buenas cosas que suceden (Peterson y Seligman, 2003). La gratitud como fortaleza relevante podría implicar una serie de beneficios para la población estudiantil, tanto individual como colectivamente, así como para la comunidad en la que se desenvuelven. La capacidad para percibir, apreciar y saborear los componentes de la propia vida se considera un factor determinante en el bienestar (Peterson et al., 2007), específicamente, la práctica de la gratitud se asocia con menos síntomas de malestar físico, valoraciones más optimistas de la vida, así como mejoramiento en el bienestar general y funcionamiento óptimo (Emmons \& McCullough, 2003; Wood, Froh y Geraghty, 2010). La gratitud se considera un rasgo de carácter tanto público como privado que motiva la reciprocidad en la interacción humana e inhibe la realización de comportamientos interpersonales destructivos (Emmons y Shelton, 2002). Algunas hipótesis con respecto a los mecanismos mediadores entre la gratitud y el bienestar se asocian con la presencia de sesgos cognitivos, estilos de afrontamiento eficientes y estados afectivos positivos (Wood et al., 2010).

En el análisis de correlaciones, la gratitud se asocia positiva y significativamente con los hábitos saludables en cuanto al consumo de alcohol, tabaco y otras drogas, lo cual podría llevar a la hipótesis de que la apreciación de la vida y lo que sucede alrededor suyo funciona como factor protector ante las opciones que presenta el consumo de sustancias (Epstein, Griffin y Botvin, 2002). Ya Logan et al. (2010) habían planteado que las fortalezas asociadas con la trascendencia juegan un papel importante en la abstinencia del consumo de sustancias. Previamente se ha planteado que el consumo de alcohol y otras drogas es utilizado por universitarios como estrategia de afrontamiento a las exigencias sociales y académicas (Grimaldo, 2012; Jiménez-Muro et al., 2009; Lema et al., 2009). De ahí que resulten relevantes los hallazgos de Wood, Joseph y Linley (2007), quienes encontraron que las personas que expresaban y sentían gratitud tenían mayores probabilidades de buscar apoyo emocional y social como medios de afrontamiento y en general tendían a usar estrategias más positivas que involucraban enfrentar los problemas (afrontamiento activo, planificación, 
reinterpretación positiva) en lugar de evitarlos (distanciamiento conductual, uso de sustancias y negación).

La curiosidad también emerge como una fortaleza presente de manera importante en la muestra de universitarios encuestados. A esta característica se le reconoce su contribución a los estados de bienestar en tanto facilita el involucramiento activo en la búsqueda del disfrute en la vida cotidiana (Kashdan y Fincham, 2004). En este sentido, se le considera una "metahabilidad" (Nakamura y Csikszentmihalyi, 2002), entendida como el reconocimiento, la búsqueda y la autorregulación voluntaria de oportunidades novedosas y estimulantes. La curiosidad implica valorar los propios intereses y contar con motivación intrínseca y autodeterminación (Kashdan y Fincham, 2004). Así, esta fortaleza posibilitaría que los estudiantes desempeñen sus actividades integrando el disfrute y sin necesidad marcada de motivaciones extrínsecas.

Por otra parte, puede notarse que tres de las cinco fortalezas con menores puntajes corresponden a aquellas relacionadas con la templanza (humildad, autorregulación y clemencia). Se ha señalado que la modestia aparece como una de las fortalezas menos prevalentes en diversas poblaciones y una de las que menos se asocia con la satisfacción vital (Park et al., 2004). Si bien el amor por el saber es altamente valorado a nivel educativo y social, tampoco presenta correlaciones significativas con la satisfacción con la vida (Park et al., 2004). Podría plantearse que, en el caso de los estudiantes universitarios participantes en esta investigación, la ubicación de estas fortalezas no afectaría su percepción de encontrarse a gusto con su vida y condiciones actuales.

Con respecto a la relevancia de las fortalezas personales en aspectos académicos, se ha encontrado que la gratitud es un factor predictor de éxito académico (Park y Peterson, 2009), sin embargo, también lo son las fortalezas intelectuales (como la curiosidad y el amor por el saber) y de templanza (autorregulación, prudencia, humildad y clemencia) (Lounsbury et al., 2009; Shoshani y Slone, 2013). La fortaleza amor por el saber describe, por una parte, el modo en que una persona se relaciones con habilidades e informaciones nuevas y, por otra, el interés individual sólidamente establecido por el cual una persona se relaciona con contenidos específicos. Las personas con amor por el saber son cognitivamente activas y típicamente experimentan sentimientos positivos en el proceso de adquirir nuevas habilidades e incrementar el conocimiento que poseen (Cosentino, 2009). Podría plantearse entonces la hipótesis de que las personas con mayores puntajes en esta fortaleza se involucren voluntariamente en conductas que conducen a obtener mejores notas (indicador común del rendimiento académico), tales como asistir activamente a clases, estudiar los materiales asignados y preocuparse por aprender los conceptos clave de las asignaturas (Lounsbury et al., 2009). La relación entre fortalezas personales y rendimiento académico podría constituirse en una vertiente de investigación en la población estudiada, aportando conocimiento relevante en el contexto de las universidades.

Los resultados relacionados con el consumo de sustancias presentan una población estudiantil con hábitos que no ponen en riesgo su salud y no se identifican diferencias por género, carrera o nivel. Es posible que el contexto en el que se desenvuelven estos estudiantes (una unidad académica en la que la promoción de la salud es central) haya contribuido al desarrollo de creencias que cumplan con un papel protector y esto se evidencia en los hábitos. La ausencia de puntuaciones que representen hábitos poco saludables o no saludables en esta 
investigación contrasta con otros estudios donde estas categorías representan el $50.1 \%$ (Salazar y Arrivillaga, 2004) y $16.6 \%$ (Arguello et al., 2009).

En cuanto a los hábitos de sueño, aparece una minoría de estudiantes con hábitos poco saludables, mientras que el resto expresa llevar a cabo prácticas que se ubican como muy saludables o saludables. Los hábitos poco saludables pueden deberse a diversos factores, tales como condiciones poco adecuadas para el descanso, exigencias académicas y laborales y dificultades de organización (Gaultney, 2010). El sueño contribuye al estado de salud en la que medida en que permite al organismo recuperar la energía vital requerida; la falta de sueño repercute en la capacidad de concentración, el estado anímico, el tiempo de reacción, el rendimiento físico e intelectual (Domínguez y Díaz, 2006; Oblitas, 2008), por lo que resulta conveniente prestar atención a posibles factores de riesgo que pudieran precipitar dificultades mayores en este ámbito.

En general, los resultados indican que la población estudiada se caracteriza por la práctica de hábitos predominantemente saludables y fortalezas personales que facilitarían su desempeño como profesionales en el ámbito de la salud. Resultaría relevante llevar a cabo estudios de corte longitudinal que permitan reconocer eventuales cambios en las variables investigadas durante el proceso de formación profesional. La información generada en esta investigación podría tomarse como base para la generación de procesos de intervención con la población estudiantil y docente, en el entendido de que las fortalezas personales constituyen aspectos fundamentales de la vivencia cotidiana de los seres humanos y su reconocimiento y puesta en práctica aportaría elementos para experiencias formativas óptimas y coherentes con estilos de vida saludables.

\section{Referencias Bibliográficas}

Arguello, M., Bautista, Y., Carvajal, J., De Castro, K., Díaz, D., Escobar, M. y Agudelo, D. (2009). Estilos de vida en estudiantes del área de la salud de Bucaramanga. Revista de Psicología Universidad de Antioquia, 1(2), 27-41. Recuperado de http://aprendeenlinea.udea.edu.co/revistas/index.php/psicologia/article/viewArticle/10 $\underline{019}$

Arrivillaga, M. y Salazar, I. C. (2005). Creencias relacionadas con el estilo de vida de jóvenes latinoamericanos. Psicología Conductual, 13(1), 19-36. Recuperado de http://www.funveca.org/revista/pedidos/product.php?id_product=217

Arrivillaga, M., Salazar, I. C. y Correa, D. (2003). Creencias sobre la salud y su relación con las prácticas de riesgo o de protección en jóvenes universitarios. Colombia Médica, 34(4), 186-195. Recuperado de https://tspace.library.utoronto.ca/handle/1807/3464

Brdar, I., \& Kashdan. T. B. (2010). Character strengths and well-being in Croatia: An empirical investigation of structure and correlates. Journal of Research in Personality, 44, 151-154. http://dx.doi.org/10.1016/j.jrp.2009.12.001

Chida, Y., \& Steptoe, A. (2008). Positive psychological well-Being and mortality: A quantitative review of prospective observational studies. Psychosomatic Medicine,70, 741-756. http://dx.doi.org/10.1097/PSY.0b013e31818105ban 
Cosentino, A. y Castro Solano, A. (2008). Inventario de virtudes y fortalezas. Manuscrito no publicado.

Cosentino, A. C. (2009). Evaluación de las virtudes y fortalezas humanas en población de habla hispana. Psicodebate, 10, 53-71. Recuperado de http://www.palermo.edu/cienciassociales/publicaciones/pdf/psico10/10Psico_03.pdf

Cosentino, A. C., \& Castro Solano, A. (2012). Character strengths: A study of Argentinean soldiers. The Spanish Journal of Psychology, 15(1), 199-215. http://dx.doi.org/10.5209/rev_SJOP.2012.v15.n1.37310

Domínguez, L. y Díaz, E. (2006). La Evaluación de los trastornos del sueño. En V. E. Caballo (Ed.), Manual para la evaluación clínica de los trastornos psicológicos. Trastornos de la edad adulta e informes psicológicos (pp.281-297). Madrid: Pirámide.

Emmons, R. A., \& McCullough, M. E. (2003). Counting blessings versus burdens: An experimental investigation of gratitude and subjective well-Being in daily life. Journal of Personality and Social Psychology, 84, 377-389. http://dx.doi.org/10.1037/00223514.84.2.377

Emmons, R A., \& Shelton, C. M. (2002) Gratitude and the science of positive psychology. En: C.R. Snyder \& S.J. Lopez (eds.), Handbook of Positive Psychology (pp. 459-471). New York: Oxford University Press.

Epstein, J. A., Griffin, K. W., \& Botvin, G. J. (2002). Positive impact of competence skills and psychological wellness in protecting inner-city adolescents from alcohol use. Prevention Science, 3(2), 95-104. http://dx.doi.org/10.1023/A:1015479216401

Gaultney, J. (2010). The prevalence of sleep disorders in college students: Impact on academic performance. Journal of American College Health, 59(2), 91-97. http://dx.doi.org/10.1080/07448481.2010.483708

Gillham, J., Adams-Deutsch, Z., Werner, J., Reivich, K., Coulter-Heindl, V., Linkins, M.,... \&Seligman, M. E. P. (2011). Character strengths predict subjective well-being during adolescence. The Journal of Positive Psychology, 6(1), 31-44. http://dx.doi.org/10.1080/17439760.2010.536773

Giménez Hernández, M. (2010). La medida de las fortalezas psicológicas en adolescentes (VIA-Youth): Relación con clima familiar, psicopatología y bienestar psicológico. (Tesis sin publicar de Doctorado en Psicología). Universidad Complutense de Madrid, Madrid. Recuperado de http://eprints.ucm.es/11578/1/T32253.pdf

Grimaldo, M. P. (2012). Estilo de vida saludable en estudiantes de posgrado de Ciencias de la Salud. Psicología y Salud, 22(1), 75-87. Recuperado de http://revistas.uv.mx/index.php/psicysalud/article/view/559/958 
Guerra de Andrade, A., Vieira Duarte, P., Pereira Barroso, L., Nishimura, R., Alberghini, D.G., \& Garcia de Oliveira, L. (2012). Use of alcohol and other drugs among Brazilian college students: Effects of gender and age. Revista Brasileira de Psiquiatria, 34, 294-305. http://dx.doi.org/10.1016/j.rbp.2012.02.002

Jiménez-Muro, A., Beamonte, A., Marqueta, A., Gargallo, P. y Nerín de la Puerta, I. (2009). Consumo de drogas en estudiantes universitarios de primer curso. Adicciones, 21(1), 21-28. Recuperado de http://www.redalyc.org/src/inicio/ArtPdfRed.jsp?iCve=289122882004

Kashdan, T. B., \& Fincham, F. D. (2004). Facilitating curiosity: A social and self-regulatory perspective for scientifically based interventions. En P. A. Linley \& S. Joseph (Eds.), Positive psychology in practice (pp. 482-503). New Jersey: Wiley \& Sons.

Kiecolt-Glaser, J. K., McGuire, L., Robles, T. R., \& Glaser, R. (2002). Emotions, morbidity, and mortality: New perspectives from psychoneuroimmunology. Annual Review of Psychology, 53, 83-107. http://dx.doi.org/10.1146/annurev.psych.53.100901.135217

Lema, L. F., Salazar, I. C., Varela, M. T., Tamayo, J. A., Rubio, A. y Botero, A. (2009). Comportamiento y salud de los jóvenes universitarios: satisfacción con el estilo de vida. Pensamiento Psicológico, 5(12), 1-88. Recuperado de http://revistas.javerianacali.edu.co/javevirtualoj/index.php/pensamientopsicologico/arti cle/view/106/316

Leontopoulou, S. \& Triliva, S. (2012). Explorations of subjective wellbeing and character strengths among a Greek University student sample. International Journal of Wellbeing, 2(3), 251-270. http://dx.doi.org/10.5502/ijw.v2.i3.6

Linley, P.A., \& Harrington, S. (2006). Playing to your strengths. The Psychologist, 19, 86-89. Recuperado de http://www.thepsychologist.org.uk/archive/archive_home.cfm/volumeID_19editionID_132-ArticleID_985-getfile_getPDF/thepsychologist/0206linl.pdf

Linley, P. A., \& Joseph, S. (2004). Applied positive psychology: A new perspective for professional practice. En P. A. Linley \& S. Joseph (Eds.), Positive psychology in practice (pp. 3-12). New Jersey: Wiley \& Sons.

Logan, D. E., Kilmer, J. R., \& Marlatt, G. A. (2010). The virtuous drinker: Character virtues as correlates and moderators of college student drinking and consequences. Journal of American College Health, 58(4), 317-324. http://dx.doi.org/10.1080/07448480903380326

Lounsbury, J. W., Fisher, L. A., Levy, J. J. \& Welsh, D. P. (2009). An investigation of character strengths in relation to the academic success of college students. Individual Differences Research, 7(1), 52-69. 
Lange, I. y Vio, F. (2006) Guía para Universidades Saludables y otras Instituciones de Educación Superior. Santiago: OPS, Universidad de Chile, Pontificia Universidad Católica de Chile. Recuperado de http://www7.uc.cl/ucsaludable/img/guiaUSal.pdf

Macaskill, A. (2012). A feasibility study of psychological strengths and well-being assessment in individuals living with recurrent depression. The Journal of Positive Psychology, 7(5). 372-386. http://dx.doi.org/10.1080/17439760.2012.702783

Maddux, J. E. (2002). Stopping the "madness". Positive psychology and the deconstruction of the illness ideology and the DSM. En: C. R. Snyder \& S. J. Lopez (eds.), Handbook of Positive Psychology (pp. 13-25). New York: Oxford University Press.

Muñoz, M. y Cabieses, B. (2008). Universidades y promoción de la salud: ¿cómo alcanzar el punto de encuentro?. Revista Panamericana de Salud Pública, 24(2), 139-146. http://dx.doi.org/10.1590/S1020-49892008000800009

Nakamura, J., \& Csikszentmihalyi, M. (2002). The concept of flow. En: C. R. Snyder \& S. J. Lopez (eds.), Handbook of Positive Psychology (pp. 89-105). New York: Oxford University Press.

Oades, L. G., Robinson, P. Green, S., \& Spence, G. B. (2011). Towards a positive university. The Journal of Positive Psychology, 6 (6), 432-439. http://dx.doi.org/10.1080/17439760.2011.634828

Oblitas, L. A. (2008). Psicología de la salud: una ciencia del bienestar y la felicidad. Avances en Psicología, 16(1), 9-38. Recuperado de http://www.incosame.com.mx/uploads/13/11/docu_5296a13ae63a0.pdf

Park, N., \& Peterson, C. (2008). The cultivation of character strengths. En: M. Ferrari and G. Potworowski (eds.), Teaching for wisdom. Cross-cultural perspectives on fostering wisdom (pp 59-77). Netherlands: Springer Science+Business Media BV.

Park, N., \& Peterson, C. (2009). Character strengths: Research and practice. Journal of College \& Character, 10(4), 1-10. http://dx.doi.org/10.2202/1940-1639.1042

Park, N., Peterson, C., \& Seligman. M. E. P. (2004). Strengths of character and well-being. Journal of Social and Clinical Psychology, 23 (5), 603-619. http://dx.doi.org/10.1521/jscp.23.5.603.50748

Park, N., Peterson, C., \& Seligman. M. E. P. (2006). Character strengths in fifty-four nations and the fifty US states. The Journal of Positive Psychology, 1(3), 118-129. http://dx.doi.org/10.1080/17439760600619567

Pedrals, N., Rigotti, A. y Bitrán, B. (2011). Aplicando psicología positiva en educación médica. Revista Médica de Chile, 139, 941-949. http://dx.doi.org/10.4067/S0034$\underline{98872011000700018}$ 
Peterson, C. (2006). A primer in positive psychology. New York: Oxford University Press.

Peterson, C., \& Park, N. (2004). Classification and measurement of character strengths: Implications for practice. En P. A. Linley \& S. Joseph (Eds.), Positive psychology in practice (pp. 433-446). New Jersey: Wiley \& Sons.

Peterson, C., Ruch, W., Beermann, U., Park, N., \& Seligman, M. E. P. (2007). Strengths of character, orientations to happiness and life satisfaction. The Journal of Positive Psychology, 2(3), 149-156. http://dx.doi.org/10.1080/17439760701228938

Peterson, C. \& Seligman, M. E. P. (2003). The Values in Action (VIA) classification of strengths. Washington, D. C.: American Psychological Association.

Primack, B. A., Kim, K. H., Shensa, A., Sidani, J. E., Barnett, T. E., \& Switzer, G. E. (2012). Tobacco, marijuana, and alcohol use in university students: A cluster analysis. Journal of American College Health, 60(5), 374-386. http://dx.doi.org/10.1080/07448481.2012.663840

Proctor, C., Maltby, J., \& Linley, P. A. (2011). Strengths use as a predictor of well-being and health-related quality of life. Journal of Happiness Studies, 12, 153-169. http://dx.doi.org/10.1007/s10902-009-9181-2

Roberts, M. C., Brown, K. J., Johnson, R. J., \& Reinke, J. (2002). Positive psychology for children. Development, prevention and promotion. En: C. R. Snyder \& S. J. Lopez (eds.), Handbook of Positive Psychology (pp. 663-675). New York: Oxford University Press.

Roth, E. (1990). Aplicaciones comunitarias de la medicina conductual. Revista Latinoamericana de Psicología, 22 (1), 27-50. Recuperado de http://redalyc.uaemex.mx/pdf/805/80522103.pdf

Salazar, I. C. y Arrivillaga, M. (2004). El consumo de alcohol, tabaco y otras drogas, como parte del estilo de vida de jóvenes universitarios. Revista Colombiana de Psicología, 13, 74-89. Recuperado de http://dialnet.unirioja.es/servlet/articulo?codigo=3246386

Sanabria, P. A., González, L. A. y Urrego, D. Z. (2007). Estilos de vida saludable en profesionales de la salud colombianos. Estudio exploratorio. Revista Med,15(2), 207217. Recuperado de http://www.scielo.org.co/scielo.php?script=sci_arttext\&pid=S0121$\underline{52562007000200008}$

Seligman, M. (2008). Positive health. Applied Psychology: An International Review, 57, 3-18. http://dx.doi.org/10.1111/j.1464-0597.2008.00351.x

Seligman, M., \& Csikszentmihalyi, M. (2000). Positive psychology. An introduction. American Psychologist, 55(1), 5-14. http://dx.doi.org/10.1037//0003-066X.55.1.5 
Seligman, M. E. P., Ernst, R. M., Gillham, J., Reivich, K., \& Linkins, M. (2009). Positive education: Positive psychology and classroom interventions. Oxford Review of Education, 35(3), 293-311. http://dx.doi.org/10.1080/03054980902934563

Sheldon, K. M., \& King, L. (2001). Why positive psychology is necessary. American Psychologist, 56, 216-217. http://dx.doi.org/10.1037//0003-066X.56.3.216

Shimai, S., Otake, K., Park, N., Peterson, C., \& Seligman, M. E. P. (2006). Convergence of character strengths in American and Japanese young adults. Journal of Happiness Studies, 7, 311-322. http://dx.doi.org/10.1007/s10902-005-3647-7

Shoshani, A., \& Slone, M. (2013). Middle school transition from the strengths perspective: Young adolescents' character strengths, subjective well-being, and school adjustment. Journal of Happiness Studies, 14(4), 1163-1181. http://dx.doi.org/10.1007/s10902$\underline{012-9374-y}$

Sing, C. Y., \& Wong, W. S. (2010). Prevalence of insomnia and its psychosocial correlates among college students in Hong Kong. Journal of American College Health, 59(3), 174-182. http://dx.doi.org/10.1080/07448481.2010.497829

Steptoe, A., Dockray, S., \& Wardle, J. (2009). Positive affect and psychobiological processes relevant to health. Journal of Personality, 77(6), 1747-1776. http://dx.doi.org/10.1111/j.1467-6494.2009.00599.x

Vázquez, C., Hervás, G., Rahona, J.J. y Gómez, D. (2009). Bienestar psicológico y salud: aportaciones desde la Psicología Positiva. Anuario de Psicología Clínica y de la Salud, 5, 15-28. Recuperado de http://institucional.us.es/apcs/doc/APCS_5_esp_15-28.pdf

Veenhoven, R. (2008). Healthy happiness: Effects of happiness on physical health and the consequences for preventive health care. Journal of Happiness Studies, 9, 449-469. http://dx.doi.org/10.1007/s10902-006-9042-1

Wood, A. M., Froh, J. J., \& Geraghty, A.W. A. (2010). Gratitude and well-being: A review and theoretical integration. Clinical Psychology Review, 30, 890-905. http://dx.doi.org/10.1016/i.cpr.2010.03.005

Wood, A. M., Joseph, S., \& Linley, P. A. (2007). Coping style as a psychological resource of grateful people. Journal of Social and Clinical Psychology, 26(9), 1076-1093. http://dx.doi.org/10.1521/jscp.2007.26.9.1076

Recepción: 05 de junio del 2014.

Corrección: 19 de enero del 2015.

Aceptación: 20 de enero del 2015.

Publicación: 31 de enero del 2015. 